\title{
EFFECTS OF WAVE-PARTICLE INTERACTIONS ON DIFFUSIVE SHOCK ACCELERATION AT SUPERNOVA REMNANTS
}

\author{
Hyesung Kang \\ Department of Earth Sciences, Pusan National University, Pusan 609-735, Korea \\ E-mail : hskang@pusan.ac.kr \\ (Received January 30, 2013; Revised February 12, 2013; Accepted February 13, 2013)
}

\begin{abstract}
Nonthermal radiation from supernova remnants (SNRs) provides observational evidence and constraints on the diffusive shock acceleration (DSA) hypothesis for the origins of Galactic cosmic rays (CRs). Recently it has been recognized that a variety of plasma wave-particle interactions operate at astrophysical shocks and the detailed outcomes of DSA are governed by their complex and nonlinear interrelationships. Here we calculate the energy spectra of CR protons and electrons accelerated at Type Ia SNRs, using time-dependent, DSA simulations with phenomenological models for magnetic field amplification due to CR streaming instabilities, Alfvénic drift, and free escape boundary. We show that, if scattering centers drift with the Alfvén speed in the amplified magnetic fields, the CR energy spectrum is steepened and the acceleration efficiency is significantly reduced at strong CR modified SNR shocks. Even with fast Afvénic drift, DSA can still be efficient enough to develop a substantial shock precursor due to CR pressure feedback and convert about 20-30\% of the SN explosion energy into CRs. Since the high energy end of the CR proton spectrum is composed of the particles that are injected in the early stages, in order to predict nonthermal emissions, especially in X-ray and $\gamma$-ray bands, it is important to follow the time dependent evolution of the shock dynamics, CR injection process, magnetic field amplification, and particle escape. Thus it is crucial to understand the details of these plasma interactions associated with collisionless shocks in successful modeling of nonlinear DSA.
\end{abstract}

Key words : cosmic ray acceleration — supernova remnant — hydrodynamics — methods: numerical

\section{INTRODUCTION}

Blast waves driven by supernova explosions are thought to produce most of Galactic cosmic rays (CRs) via diffusive shock acceleration (DSA), at least up to the first knee energy of $10^{15.5} \mathrm{eV}$ and possibly to the second knee energy of $10^{17.5} \mathrm{eV}$ (e.g., Blandford \& Eichler 1987; Hillas 2005; Reynolds 2008). In DSA theory, suprathermal particles are scattered by magnetohydrodynamic (MHD) waves and accelerated to high energies through multiple crossings of a collisionless shock in tenuous astrophysical plasmas (Bell 1978; Drury 1983; Malkov \& Drury 2001). Nonlinear treatments of DSA predict that, if more than $10^{-4}$ of incoming particles are injected into the CR population, order of $10 \%$ of the explosion energy can be transferred to CRs at supernova remnant (SNR) shocks, which is sufficient enough to replenish the CR energy escaping from the Galaxy (e.g., Berezhko et al. 2009, 2012; Kang 2006, 2010).

As a result of nonlinear CR feedback, a smooth precursor should develop upstream of the strong SNR shock, and the ensuing CR proton spectrum just below the high energy cutoff is predicted to be flatter than the test-particle power-law of $N(E) \propto E^{-2}$. Such a flat CR proton spectrum leads to a $\gamma$-ray photon spectrum from $\pi^{0}$ decay harder than $N\left(E_{\gamma}\right) \propto E_{\gamma}^{-2}$. On the contrary, the observed $\gamma$-ray spectra in $\mathrm{GeV}-\mathrm{TeV}$ band of young
SNRs seem to be much softer, i.e., $N\left(E_{\gamma}\right) \propto E_{\gamma}^{-\Gamma}$ with $2 \lesssim \Gamma \lesssim 2.7$ (Abdo et al. 2010; Acero et al. 2010; Acciari et al. 2011; Caprioli 2011; Giordano et al. 2012; Mandelartz \& Tjus 2013). Thus the observed $\gamma$-ray spectrum of SNRs appears to be inconsistent with the flat CR proton spectrum predicted by nonlinear DSA model, which may call for a significant revision of the standard DSA theory.

X-ray continuum observed in thin rims of several young Galactic SNRs including Tycho, Kepler and SN1006 can be interpreted as synchrotron emission from $10-100 \mathrm{TV}$ electrons cooling radiatively in the magnetic field as strong as a few $100 \mu \mathrm{G}$ downstream of the forward shock (e.g., Bamba et al. 2003; Parizot et al. 2006; Eriksen et al. 2011). This indicates that the magnetic field is effectively enhanced at strong SNR shocks, since the mean interstellar magnetic field is about 5-8 $\mu \mathrm{G}$ (Völk et al. 2005; Reynolds et al. 2012).

Indeed, it has been shown that at a strong CR dominated shock the CRs streaming ahead of the shock excite both resonant and non-resonant waves on scales comparable to or smaller than the CR gyroradius $\left(r_{g}\right)$, and amplify the turbulent magnetic fields by orders of magnitude in the precursor (e.g., Bell 1978; Lucek \& Bell 2000; Bell 2004; Riquelme 
\& Spitkovsky 2009, 2010; Gargaté et al. 2010). Furthermore, it has been suggested that magnetic fluctuations on scales larger than the CR gyroradius can grow through mean-field dynamo effects in the presence of small-scale, circularly-polarized Alfvén waves (Bykov et al. 2011; Rogachevskii et al. 2012), and possibly through other instabilities including the firehose, filamentation, and acoustic instabilities (e.g., Beresnyak et al. 2009; Drury \& Downes 2012; Schure et al. 2012; Caprioli \& Spitkovsky 2012; Reville \& Bell 2012). So magnetic field amplification (MFA) should be regarded as an integral part of nonlinear DSA at strong collisionless shocks.

It was recognized early on that the excited Alfvén waves are to drift with respect to the background flow $(\mathbf{u})$, so the mean convective velocity of the scattering centers becomes $\mathbf{u}+\mathbf{u}_{\mathbf{w}}$, where $\mathbf{u}_{\mathbf{w}}$ is the mean drift speed of the scattering centers relative to the local gas flow (Skilling 1975; Bell 1978). Because of the negative gradient of the $\mathrm{CR}$ pressure in the shock precursor, the backward waves moving against the background flow dominate in the upstream region, so the upstream wave drift speed is $u_{w, 1} \approx+v_{A}$ (Bell 1978), where $v_{A}=B / \sqrt{4 \pi \rho}$ is the local Alfvén speed. It is not well known, however, in the downstream region, if the forward and backward moving waves are nearly balanced (i.e., $u_{w, 2} \approx 0$ ) or the forward waves dominate due to the positive gradient of the $\mathrm{CR}$ pressure behind the shock (i.e., $u_{w, 2} \approx-v_{A}$ ). In either case, as a result of the wave drift, the compression ratio that the scattering centers experience across the shock becomes smaller than that of the underlying flow (Bell 1978; Schlickeiser 1989). Since the particles are isotropized in the mean wave frame, the resulting CR spectrum becomes much softer than predicted with the velocity jump for the background flow (Caprioli et al. 2009; Kang 2010). So phenomenological models for self-consistent treatments of MFA and Alfvénic drift have been implemented in nonlinear DSA simulations of SNRs in effort to obtain the CR proton spectrum softer than the canonical test-particle power-law of $E^{-2}$ (e.g.. Vladimirov et al. 2008; Zirakashvili \& Ptuskin 2008, 2012; Lee et al. 2012; Caprioli 2012; Kang 2012).

In addition, geometrical effects of expanding spherical shocks, time-varying injection process and escape of CRs from SNRs can soften the CR spectrum near the high energy cut-off (Drury 2011). The highest energy particles should escape from the shock as the SNR slows down in the late Sedov-Taylor stage due to the following reasons: 1) The diffusion length of CRs becomes greater the shock curvature radius, i.e., $\kappa\left(p_{\max }\right) / u_{s}(t) \gtrsim \zeta r_{s}(t)$, where $\kappa(p)$ is the CR diffusion coefficient and $\zeta \sim 0.1-1$ is a dimensionless parameter (e.g., Caprioli et al. 2010). 2) Non-linear wave damping and wave dissipation due to ion-neutral collisions may weaken stochastic scattering on relevant scales (Ptuskin \& Zirakashvili 2005; Malkov et al. 2011). Since the escape process depends on the timedependent history of the shock properties such as $u_{s}$, $r_{s}$, and $B$ as well as the MHD turbulence spectrum, the problem becomes very complex (Drury 2011).

Multi-band observations of nonthermal emissions from radio to $\gamma$-ray have provided a powerful tool to examine theoretical models of nonlinear DSA at SNRs (e.g., Berezhko \& Völk 1997; Berezhko et al. 2009, 2012; Caprioli 2011; Edmon et al. 2011). For example, radio spectral index carries the information on the power-law portion of $\sim \mathrm{GeV}$ electrons, while X-ray continuum reflects the exponential cut-off near the maximum electron energy, $E_{\mathrm{e}, \max } \sim 10 \mathrm{TeV}$, and the magnetic field strength. Because of the balance between DSA energy gains and radiative losses, $E_{\text {e,max }}$ depends mainly on the evolution of $u_{s}(t)$ and $B(r, t)$. For highly amplified magnetic fields, the maximum electron energy should decrease due to radiative cooling, while the maximum proton energy is expected to increase in time. The interpretation of observed $\gamma$-ray spectrum is more complicated, since $\gamma$-ray emissions can originate from both CR protons and CR electrons: the decay of neutral pions produced in nuclear interactions between CR protons and the background medium, inverse Compton (iC) scattering of the background radiation by CR electrons, and electronic nonthermal bremsstrahlung. Obviously, the relative importance of these different components is governed by several factors such as the CR particle spectra at the high energy end (both $p_{\mathrm{e}, \max }$ and $\left.p_{\mathrm{p}, \max }\right)$, the magnetic field strength, the background gas density, $n_{H, 0}$, and the background radiation field, and the electron to proton ratio, $K_{e / p}$. So MFA, Alfvénic drift and escape of the highest energy particles should be considered self-consistently in order to estimate accurately nonthermal radiation spectrum of SNRs.

Wave-particle interactions relevant to collisionles shocks can be studied from first principles, using particle-in-cell (PIC) simulations (e.g., Riquelme \& Spitkovsky 2009, 2010; Ohira et al. 2009) and hybrid plasma simulations (e.g., Lucek \& Bell 2000; Giacalone \& Jokipii 2007; Guo et al. 2012; Gargaté \& Spitkovsky 2012). In PIC simulations, the Maxwell's equations for electric and magnetic fields are solved along with the equations of motion for ions and electrons, so the full wave-particle interactions can be followed from first principles. But extremely wide ranges of length and time scales need to be covered mainly because of the large ratio of proton to electron mass. In hybrid simulations, only the ions are treated kinetically while the electrons are treated as a neutralizing, massless fluid, which reduces the severe computational requirements. However, it is still prohibitively expensive to simulate the full extent of DSA from the thermal energies of background plasma to the relativistic energies of CRs, following the relevant plasma interactions at the same time. So we do not yet have full understandings of injection and diffusive scattering of CRs and MFA to make detailed quantitative predictions for nonlinear DSA. Instead, most of kinetic DSA approaches, in which the diffusion-convection equation for 
the phase-space distribution of particles is solved, commonly adopt phenomenological models that may emulate some of those processes (e.g., Kang et al. 2002; Berezhko et al. 2009; Ptuskin et al. 2010; Lee et al. 2012; Caprioli 2012; Kang 2012).

In the present paper, we performed time-dependent numerical simulations, in which DSA of CR protons and electrons at the forward shock of Sedov-Taylor SNRs is followed along with electronic synchrotron and $\mathrm{iC}$ losses. In order to explore how wave-particle interactions affect the outcomes of DSA, phenomenological models for MFA due to CR streaming instabilities and Alfvénic drift are implemented. Escape of the highest energy protons near $p_{\mathrm{p} \text {, max }}$ is also considered by adopting a free escape boundary (FEB) at an upstream location. As in our previous works (e.g., Kang 2010, 2012), here we adopt simple models for thermal leakage injection and Bohm-like diffusion $(\kappa(p) \propto p)$. In the next section we describe the numerical method and phenomenological models for the plasma interactions in DSA theory, and the model parameters for SedovTaylor blast wave. The simulation results will be discussed in Section 3, followed by a summary in Section 4.

\section{DSA MODEL}

\subsection{CRASH Code for DSA}

Here we consider the $\mathrm{CR}$ acceleration at quasiparallel shocks where the uniform background magnetic field, $\mathbf{B}_{\mathbf{0}}$, is parallel to the flow velocity, $\mathbf{u}$. In the diffusion approximation, where the pitch-angle distribution of CRs is nearly isotropic, the Fokker-Plank equation is reduced to the following diffusion-convection equation: (Skilling 1975):

$$
\begin{array}{r}
\frac{\partial g}{\partial t}+\left(u+u_{w}\right) \frac{\partial g}{\partial r}=\frac{1}{3 r^{2}} \frac{\partial}{\partial r}\left[r^{2}\left(u+u_{w}\right)\right]\left(\frac{\partial g}{\partial y}-4 g\right) \\
+\frac{1}{r^{2}} \frac{\partial}{\partial r}\left[r^{2} \kappa(r, y) \frac{\partial g}{\partial r}\right]+p \frac{\partial}{\partial y}\left(\frac{b}{p^{2}} g\right)
\end{array}
$$

where $g_{p}=f_{p} p^{4}$ and $g_{e}=f_{e} p^{4}$ are the pitch-angleaveraged phase space distribution functions for CR protons and electrons, respectively, $y=\ln \left(p / m_{p} c\right)$ is the logarithmic momentum variable, and $\kappa(r, y)$ is the spatial diffusion coefficient along the direction parallel to $\mathbf{B}_{\mathbf{0}}$. The wave drift velocity $u_{w}$ represents the effective relative motion of scattering centers with respect to the bulk flow velocity, which will be described in detail in Section 2.3.

The electronic synchrotron and inverse Compton (iC) cooling constant is defined as $b(p)=-d p / d t=$ $\left(4 e^{4} / 9 m_{e}^{4} c^{6}\right) B_{\mathrm{e}}^{2} p^{2}$ in cgs units, where $e$ and $m_{e}$ are electron charge and mass, respectively. Here $B_{\mathrm{e}}^{2}=B^{2}+B_{r}^{2}$ as the effective magnetic field strength for radiative losses including the energy density of the ambient radiation field. We set $B_{r}=6.5 \mu \mathrm{G}$, including the cosmic background and mean Galactic radiation fields (Edmon et al. 2011). The cooling term is set to be $b(p)=0$ for protons.

The flow velocity, $u$, is defined in the rest frame of the ambient medium and calculated by solving the momentum conservation equation with dynamical feedback of the CR pressure and self-generated magnetic fields,

$$
\frac{\partial(\rho u)}{\partial t}+\frac{\partial}{\partial r}\left(\rho u^{2}+P_{g}+P_{c}+P_{B}\right)=-\frac{2}{r} \rho u^{2} .
$$

The CR pressure, $P_{c}$, is calculated self-consistently with the $\mathrm{CR}$ proton distribution function, $f_{p}(r, p)$, while the magnetic pressure, $P_{B}$, is calculated according to our phenomenological model for MFA (see Section 2.2). The dynamical effects of the CR electron pressure are negligible. The basic gasdynamic equations and details of the spherical CRASH (Cosmic-Ray Amr SHock) code can be found in Kang \& Jones (2006).

\subsection{Magnetic Field Amplification}

Caprioli (2012) has shown that for strong shocks with $M_{s} \gg 1$, the turbulent magnetic field amplified by CR streaming instabilities can be approximated as

$$
\frac{\delta B^{2}}{8 \pi \rho_{0} u_{s}^{2}}=\frac{2}{25} \frac{\left(1-U^{5 / 4}\right)^{2}}{U^{3 / 2}}
$$

where $U(r)=\left[u_{s}-|u(r)|\right] / u_{s}$ is the flow speed in the shock rest frame normalized by the shock speed. This was derived by assuming that the amplified magnetic fields are isotropized and so the effective Alfvén speed is defined by the local amplified field. The first assumption for isotropic fields seems to be justified according to the recent hybrid MHD simulation study by Reville \& Bell (2013), which has demonstrated that selfgenerated magnetic fields in the precursor of strong CR modified shocks are highly disorganized. So we adopt the following recipe in the upstream region $\left(r>R_{s}\right)$,

$$
\frac{B(r)^{2}}{B_{0}^{2}}=1+\left(1-\omega_{H}\right) \cdot \frac{4}{25} M_{A, 0}^{2} \frac{\left(1-U(r)^{5 / 4}\right)^{2}}{U(r)^{3 / 2}}
$$

where $M_{A, 0}=u_{s}(t) / v_{A, 0}$ is the Alfvénic Mach number for the instantaneous shock speed with respect to the Alfvén speed, $v_{A, 0}=B_{0} / \sqrt{4 \pi \rho_{0}}$, in the far upstream region. The factor $\left(1-\omega_{H}\right)$ is introduced to account for the wave dissipation and ensuing reduction of MFA, which will be discussed in Section 2.4. Apparently, $\omega_{H}=0$ means no wave dissipation, while $\omega_{H}=1$ means complete dissipation and no MFA. Here $\omega_{H}=0.5$ will be considered as a fiducial case.

Note that this recipe is intended to be a heuristic model that may represent qualitatively the MFA process in the shock precursor. It predicts no MFA in the test-particle regime, in which the flow structure is not modified, i.e., $U(r)=1$. In the case of "moderately modified" shocks, for example, if the normalized velocity immediately upstream of the subshock, $U_{1} \approx 0.8$, 
Table 1. Model Parameters ${ }^{\mathrm{a}}$

\begin{tabular}{|c|c|c|c|c|c|c|c|c|c|}
\hline Model $^{b}$ & $\begin{array}{r}n_{H}(\mathrm{ISM}) \\
\left(\mathrm{cm}^{-3}\right)\end{array}$ & $\begin{array}{r}T_{0} \\
(\mathrm{~K})\end{array}$ & $\begin{array}{r}r_{o} \\
\text { (pc) }\end{array}$ & $\begin{array}{r}t_{o} \\
\text { (years) }\end{array}$ & $f_{A}{ }^{\mathrm{c}}$ & $\omega_{H}{ }^{\mathrm{d}}$ & $\zeta^{\mathrm{e}}$ & $\begin{array}{r}u_{w, 2}{ }^{\mathrm{f}} \\
\left(\mathrm{km} \mathrm{s}^{-1}\right)\end{array}$ & $v_{A, 0}$ \\
\hline W1 & 0.3 & $3 \times 10^{4}$ & 3.18 & 255 . & 1.0 & 0.5 & 0.1 & 0 & 16.8 \\
\hline W2 & 0.3 & $3 \times 10^{4}$ & 3.18 & 255. & 1.0 & 0.1 & 0.1 & 0 & 16.8 \\
\hline W3 & 0.3 & $3 \times 10^{4}$ & 3.18 & 255 . & 0.1 & 0.5 & 0.1 & 0 & 16.8 \\
\hline W4 & 0.3 & $3 \times 10^{4}$ & 3.18 & 255 . & 1.0 & 0.5 & 0.1 & $-v_{A}$ & 16.8 \\
\hline $\mathrm{H} 1$ & 0.01 & $10^{6}$ & 6.62 & 792. & 1.0 & 0.5 & 0.1 & 0 & 92.2 \\
\hline $\mathrm{H} 2$ & 0.01 & $10^{6}$ & 6.62 & 792. & 1.0 & 0.1 & 0.1 & 0 & 92.2 \\
\hline H3 & 0.01 & $10^{6}$ & 6.62 & 792. & 0.1 & 0.5 & 0.1 & 0 & 92.2 \\
\hline $\mathrm{H} 4$ & 0.01 & $10^{6}$ & 6.62 & 792. & 1.0 & 0.5 & 0.1 & $-v_{A}$ & 92.2 \\
\hline W1b & 0.3 & $3 \times 10^{4}$ & 3.18 & 255 . & 1.0 & 0.5 & 0.25 & 0 & 16.8 \\
\hline W1c & 0.3 & $3 \times 10^{4}$ & 3.18 & 255. & 1.0 & 0.5 & 0.5 & 0 & 16.8 \\
\hline
\end{tabular}

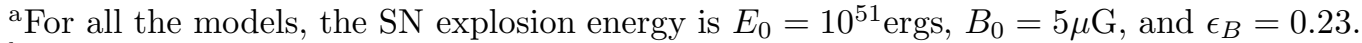

b' $\mathrm{W}$ ' and ' $\mathrm{H}$ ' stands for the warm and hot phase of the ISM, respectively.

${ }^{\mathrm{c}}$ See Eq. 8 for the Alfvén drift parameter.

${ }^{\mathrm{d}}$ See Eq. 11 for the wave dissipation parameter.

${ }^{\mathrm{e}}$ Free escape boundary is at $r_{\mathrm{FEB}}=(1+\zeta) r_{s}(t)$.

${ }^{\mathrm{f}}$ For all the models, $u_{w, 1}=+v_{A}$ is assumed for the preshock region.

and the wave dissipation parameter, $\omega_{H} \approx 0.5$, then the amplified magnetic field strength immediately upstream of the subshock scales as $B_{1} / B_{0} \approx 0.1 M_{A, 0} \propto$ $u_{s}$. Hereafter, we use the subscripts ' 0 ', ' 1 ', and ' 2 ' to denote conditions far upstream of the shock, immediately upstream and downstream of the subshock, respectively. In the case of "strongly modified" shocks (e.g., $U(r) \ll 1)$, the magnetic field energy density scales as $\left(B(r) / B_{0}\right)^{2} \propto U(r)^{-3 / 2}$ and may increase to a significant fraction of the shock kinetic energy, which is not compatible with observations (Reynolds et al. 2012). So in the simulations the amplification factor is restricted by the following condition:

$$
\left(B_{1}^{2} / 8 \pi\right) \lesssim \eta(1 / 2) \rho_{0} u_{s}^{2},
$$

where $\eta \approx 10^{-2}$ is enforced. This condition keeps the downstream magnetic energy density, $B_{2}^{2} / 8 \pi$, less than $10 \%$ of the shock kinetic energy for $\rho_{2} / \rho_{1} \sim 4$ (see Eq. 6). However, the energy condition (5) is turned on only briefly during the early stage when the shock is very strong and for the models with minimum Alfvénic drift (e.g., Models W3 and H3, see Table 1).

We then assume $B_{1}$ is completely turbulent (isotropic) and the two perpendicular components are simply compressed across the subshock. Then, the immediate postshock field strength is estimated by

$$
B_{2} / B_{1}=\sqrt{1 / 3+2 / 3\left(\rho_{2} / \rho_{1}\right)^{2}} .
$$

So for example, if $\rho_{2} / \rho_{1} \approx 4, B_{2} / B_{1} \approx 3.3$. Although the downstream magnetic field strength is expected to decrease behind a spherically expanding blast wave, the detailed configuration and the evolution of magnetic fields between the forward shock and the contact discontinuity are not well known. So we adopt a simple model, in which the magnetic fields decay behind the shock and the field strength scales with the gas density, i.e., in the downstream region $\left(r<R_{s}\right)$

$$
B(r)=B_{2} \cdot\left[\rho(r) / \rho_{2}\right] .
$$

\subsection{Alfvénic Drift}

Alfv́en waves generated by CR streaming instabilities drift with respect to the underlying flow and also transfer energy to the gas through dissipation (e.g., Skilling 1975; Jones 1993). As discussed in the Introduction, Alfvénic drift in the precursor reduces the velocity difference that the particles experience while scattering across the shock. As a result, the CR spectrum becomes softer than estimated without considering the wave drift.

The mean drift speed of scattering centers is commonly set to be the Alfvén speed, i.e., $u_{w, 1}(r)=+v_{A}=$ $+B_{\|} / \sqrt{4 \pi \rho}$ in the precursor, pointing away from the shock, where $B_{\|}$is the magnetic field parallel to the shock normal. In a strong CR modified shock, CR streaming instabilities can be strongly nonlinear, so the turbulent magnetic fields become fully disorganized and isotropic (Reville \& Bell 2013). Moreover, plasma simulations of Bell's CR current driven instability have shown that both $B_{\|} / B_{0}$ and $B_{\perp} / B_{0}$ can increase to $\sim 10-30$ (Riquelme \& Spitkovsky 2009) and the waves drift roughly with the Alfvén speed in the amplified fields (Gargaté et al. 2010). Since it is not well understood how the Alfvén speed behaves in such turbulent 
magnetic fields, we model the local Aflvén speed as

$$
v_{A}(r)=\frac{B_{0}+\left(B(r)-B_{0}\right) f_{A}}{\sqrt{4 \pi \rho(r)}},
$$

where the parameter $f_{A}$ is a free parameter ( $\mathrm{Zi}$ rakashvili \& Ptuskin 2008; Lee et al. 2012). If scattering centers drift in the unperturbed fields, then $f_{A}=0$, which limits the Alfvénic drift effects to a minimum level. Here we will consider the models with $f_{A}=0.1-1.0$ (see Table 1$)$.

In the postshock region the Alfvénic turbulence is probably relatively balanced in planar shocks, so the wave drift would be negligible, $u_{w, 2} \approx 0$ (Jones 1993). For spherical Sedov-Taylor blast waves, however, the CR pressure as well as the gas pressure and density all decrease behind the shock, so Alfven wave may drift inward with $u_{w, 2} \approx-v_{A}$ downstream of the shock. In that case, the accelerated particle spectrum could be softened drastically (e.g., Zirakashvili \& Ptuskin 2008). We will consider the models with both upstream and downstream drift (see Table 1).

As mentioned in the Introduction, the CR spectrum develops a concave curvature when the preshock flow is modified by the CR pressure at strong shocks. If we include Alfvénic drift, the slope of the momentum distribution function, $q=-\partial \ln f / \partial \ln p$, should be further modified. According to the MFA prescription given in Eq. 4, the amplification factor depends on the precursor modification, that is, the ratio $B(r) / B_{0}$ is unity far upstream and increases through the precursor toward the subshock. So the Afvénic drift speed is the highest immediately upstream of the subshock, while it is the same as the unperturbed Alfvén speed, $v_{A, 0}$ at the far upstream region $\left(M_{A, 1} \ll M_{A, 0}\right)$. Thus Alfvénic drift is expected to steepen preferentially the low energy end of the CR spectrum, since the lowest energy particles diffuse mostly near the subshock. For the highest energy particles, which diffuse over the distance of $\sim \kappa\left(p_{\mathrm{p}, \max }\right) / u_{s}$, Alfévnic drift (mostly defined with $\left.B_{0}\right)$ does not steepen the CR spectrum significantly, if $M_{A, 0} \gg 1$ (Kang 2012).

\subsection{Wave Dissipation and Particle Escape}

In the late Sedov-Taylor stage non-linear wave damping and dissipation due to ion-neutral collisions quench the MHD turbulence, leading to slower acceleration and escape of the highest energy particles from the shock. Again these processes are not well understood, so we implement a free escape boundary (FEB) at an upstream location, that is, setting $f(r, p)=0$ for $r \geq r_{\mathrm{FEB}}=(1+\zeta) r_{s}(t)$, where $\zeta=0.1-0.5$ (Caprioli et al. 2010). This FEB condition can mimic the escape of particles with the diffusion length, $\kappa(p) / u_{s}(t) \gtrsim \zeta r_{s}(t)$. Since the shock radius increases but the shock speed decreases in time, with this FEB the mean momentum of escaping particles decreases as $\left\langle p_{\text {esc }}\right\rangle \propto t^{-1 / 5}$.

The flux of escaping particles can be estimated from the diffusive flux out of FEB (Caprioli et al. 2010)

$$
\phi_{\mathrm{esc}}(t, p)=-\left[\kappa(r, p) \frac{\partial f}{\partial r}\right]_{r_{\mathrm{FEB}}} .
$$

Then the time-integrated particle spectrum escaped from the shock up to the shock age $t$ is given by

$$
\Phi_{\mathrm{esc}}(p)=\int_{t_{i}}^{t} 4 \pi r_{\mathrm{FEB}}(t)^{2} \phi_{\mathrm{esc}}(t, p) \mathrm{dt} .
$$

As mentioned above, we adopt a simple model in which Alfvén waves are dissipated locally as heat. So gas heating term in the upstream region is prescribed as

$$
W(r, t)=-\omega_{H} \cdot v_{A}(r) \frac{\partial P_{c}}{\partial r},
$$

where $\omega_{H}$ is introduced to control the degree of wave dissipation. As shown in previous studies (e.g., Berezhko \& Völk 1997; Kang \& Jones 2006; Caprioli et al. 2009), this precursor heating reduces the subshock Mach number thereby reducing the DSA efficiency.

\subsection{Thermal Leakage Injection}

The particle speed must be several times faster than the downstream flow speed in order for suprathermal particles to swim against MHD turbulence across the shock transition zone. So CRs are injected at the subshock with the injection momentum, $p_{\text {inj }} \approx$ $1.17 m_{p}\left(u_{s} / \sigma_{2}\right)\left(1+1.07 \epsilon_{B}^{-1}\right)$, where $\sigma_{2}=\rho_{2} / \rho_{0}$ is total compression ratio and $\epsilon_{B}$ is the injection parameter that regulates the injection rate (Kang et al. 2002). In an expanding SNR, the shock slows down and the subshock weakens as the precursor develops due to nonlinear feedback of the CR pressure, so the Maxwellian velocity distribution shifts to lower momenta and $p_{\text {inj }}$ decreases in time (see for example Fig. 5). With $\epsilon_{B}=0.23$ adopted here, the injected particle fraction, $\xi=n_{\mathrm{cr}, 2} / n_{2}$, varies from $10^{-5}$ to $10^{-3.5}$, which is comparable to the values commonly adopted in DSA calculations for SNRs (e.g., Berezhko et al. 2009; Morlino et al. 2009; Morlino \& Caprioli 2012). It was shown previously that for $\xi \gtrsim 10^{-4}$ the DSA efficiency saturates at strong shocks (Berezhko \& Völk 1997; Kang \& Jones 2006; Caprioli 2012), so the acceleration efficiency obtained here should be considered as an upper limit for the efficient injection regime.

For $p \geq p_{\text {inj }}$, protons and electrons of the same rigidity $(R=p c / Z e$, where $Z$ is the charge number of CRs) are injected in the same manner in the simulations. But it is expected that electrons are injected at the shock with a much smaller injection rate, i.e., the CR electron-to-proton ratio, $K_{e / p} \sim 10^{-4}-10^{-2}$, because postshock thermal electrons need to be preaccelerated (Reynolds 2008; Morlino \& Caprioli 2012). Since this ratio is not yet constrained accurately by plasma physics, $K_{e / p}$ is often treated as a free parameter that can be adjusted to obtain the best fit of nonthermal radiation spectra of observed SNRs. We set $K_{e / p}=0.1$ in some figures below just for clarity. 


\subsection{Bohm-like Diffusion Model}

In our model, turbulent MHD waves are self-generated efficiently by plasma instabilities driven by CRs streaming upstream in the shock precursor, so we can assume that $\mathrm{CR}$ particles are resonantly scattered by Alfvén wave turbulence with fully saturated spectrum. Then the particle diffusion can be approximated by a Bohmlike diffusion coefficient, $\kappa_{B} \sim(1 / 3) r_{g} v$, but with flattened non-relativistic momentum dependence:

$$
\kappa(r, p)=\kappa_{\mathrm{n}}\left(\frac{B_{0}}{B_{\|}(r)}\right) \cdot\left(\frac{p}{m_{p} c}\right),
$$

where $\kappa_{n}=m_{p} c^{3} /\left(3 e B_{0}\right)=\left(3.13 \times 10^{22} \mathrm{~cm}^{2} \mathrm{~s}^{-1}\right) B_{0}^{-1}$, and $B_{0}$ is the magnetic field strength far upstream expressed in units of microgauss. The parallel component of local magnetic field, $B_{\|}(r)$, is given by Eqs. 4-7.

\subsection{Sedov-Taylor Blast Waves}

Here we consider a Type Ia supernova explosion with the ejecta mass, $M_{e j}=1.4 M_{\odot}$, expanding into a uniform ISM. All models have the explosion energy, $E_{o}=10^{51}$ ergs. Previous studies have shown that the shock Mach number is one of the key parameter determining the evolution and the DSA efficiency (e.g., Kang 2010), so two phases of the ISM are considered: the warm phase with $n_{H}=0.3 \mathrm{~cm}^{-3}$ and $T_{0}=3 \times 10^{4} \mathrm{~K}$ (W1-W4 models), and the hot phase with $n_{H}=0.01 \mathrm{~cm}^{-3}$ and $T_{0}=10^{6} \mathrm{~K}$ (H1-H4 models). The background gas is assumed to be completely ionized with the mean molecular weight, $\mu=0.61$, and the background magnetic field strength is set to be $B_{0}=5 \mu \mathrm{G}$. For the warm ISM models the Alfvén speed is $v_{A}=16.8 \mathrm{~km} \mathrm{~s}^{-1}$, so the Alfvén Mach number is given as $M_{A, 0} \approx 180\left(u_{s} / 3000 \mathrm{~km} \mathrm{~s}^{-1}\right)$. For the hot ISM models, $v_{A}=183 \mathrm{~km} \mathrm{~s}^{-1}$ and $M_{A, 0} \approx$ $16.4\left(u_{s} / 3000 \mathrm{~km} \mathrm{~s}^{-1}\right)$. The rest of models parameters are summarized in Table 1.

We consider W1 and H1 models as fiducial cases with canonical values of model parameters. In the hot ISM models, both the sonic and Alfvénic Mach numbers are smaller, compared to the warm ISM models, so the degree of MFA and the DSA efficiency are to be much lower and the accelerated particle spectra are softer due to Alfvénic drift. W2 and H2 models have a lower dissipation rate $\left(\omega_{H}=0.1\right)$, while $\mathrm{W} 3$ and $\mathrm{H} 3$ models have a smaller Alfvén speed $\left(f_{A}=0.1\right)$. Models W4 and H4 are chosen to see the effects of Alfvénic drift in the postshock region. Models W1b and W1c are set up to study the effects of different FEB locations: $r_{\mathrm{FEB}}=1.25 r_{s}$ and $1.5 r_{s}$, respectively.

The physical quantities are normalized, both in the numerical code and in the plots below, by the following constants: $r_{o}=\left(3 M_{e j} / 4 \pi \rho_{o}\right)^{1 / 3}, t_{o}=\left(\rho_{o} r_{o}^{5} / E_{o}\right)^{1 / 2}$, $u_{o}=r_{o} / t_{o}, \rho_{o}=\left(2.34 \times 10^{-24} \mathrm{~g}\right) n_{H}$, and $P_{o}=\rho_{o} u_{o}^{2}$ (see Table 1).
During the initial free-expansion stage of Type Ia SNRs, a forward shock expands as $r_{s} \propto t$, while a reverse shock moves inward as the pressure of the central ejecta decreases due to expansion. After the swept up mass becomes considerably larger than the ejecta mass, the SNR enters the Sedov-Taylor (ST hereafter) stage during which the forward shock evolves as $r_{s} \propto t^{2 / 5}$. In fact the true ST stage is established only after the reverse shock is reflected at the center, so the dynamical evolution of real SNRs is much more complex than that of the ST similarity solution (Kang 2006).

Here we adopt the ST similarity solution, $r_{\mathrm{ST}} / r_{o}=$ $1.15\left(t / t_{o}\right)^{2 / 5}$, without the contact discontinuity and the reverse shock, since the current version of CRASH code can treat only a single shock. Although the early dynamical evolution of SNRs is not realistic in our simulations, this strategy can be justified, because our goal is to explore how wave-particle interactions included in our DSA simulations affect the high energy end of the CR spectra rather than realistic model fitting of the observed properties of a specific SNR.

For Type Ia SNRs, the highest momentum of CRs is achieved at the beginning of the ST stage and the transfer of explosion energy to the CR component occurs mostly during the early ST stage (e.g., Berezhko \& Völk 1997). In order to account for the CR acceleration from free expansion stage through ST stage, we begin the calculations with the ST similarity solution at $t / t_{o}=0.2$ and follow the evolution of the forward shock up to $t / t_{o}=10$.

The spherical grid is expanding in a comoving way with the forward shock (Kang \& Jones 2006). The inner boundary is located at $r / r_{s}=0.1$ at $t / t_{o}=0.2$, while the outer boundary coincides with the FEB. The gasdynamic variables are assumed to be continuous at the both boundaries. The $\mathrm{CR}$ distribution functions are continuous across the inner boundary, while they are set to be zero at the FEB.

\subsection{Cutoff Momenta}

It will be useful to understand how the maximum momenta for protons and electrons $\left(p_{\mathrm{p}, \max }\right.$ and $\left.p_{\mathrm{e}, \max }\right)$ that can be accelerated at SNRs depend on the shock age and other parameters. From the simple acceleration timescale argument, the maximum proton momentum is expected to increase, if the magnetic field is amplified globally. Since MFA depends on the flow modification in our model, however, the upstream magnetic field asymptotes to $B_{0}$ at the FEB, even though it increases to $B_{1}$ immediately upstream of the subshock (see Fig. 4 below). Considering that the highest energy particles diffuse over the entire precursor, the mean residence time of particles with $p_{\mathrm{p}, \max }$ in the upstream side should be close to $\tau_{1} \approx \kappa_{n} p_{\mathrm{p}, \max } / u_{0}$ and remain similar to that for the case with $B_{0}$. The mean residence time in the downstream side, $\tau_{2} \approx\left(\kappa_{n} p_{\mathrm{p}, \max } / u_{0} \sigma_{2}\right)\left(B_{0} / B_{2}\right)$, is significantly reduced for the case of strong MFA $\left(B_{2} \gg B_{0}\right)$. As a result, the mean acceleration time, 
DIFFUSIVE SHOCK ACCELERATION AT SNRS

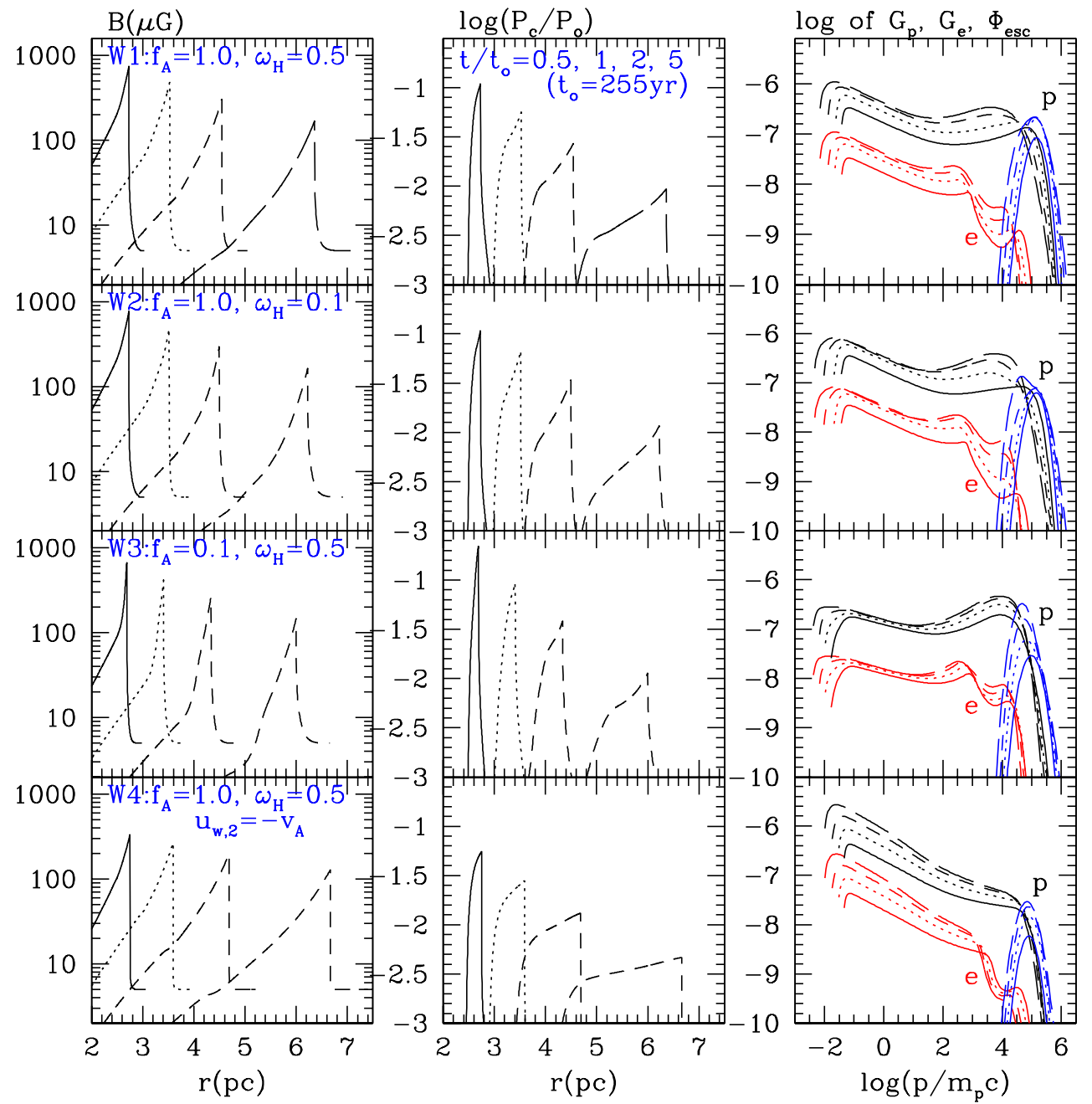

Fig. 1. - Time evolution of W1-W4 models at $t / t_{o}=0.51$ (solid lines), 1 (dotted), 2 (dashed) and 5 (long dashed). See Table 1 for model parameters and normalization constants. In the left column, magnetic fields are given in units of microgauss, while the $\mathrm{CR}$ pressure is expressed in terms of $P_{o}$ in the middle column. In the right column the volume integrated distribution function, $G_{p}(p)$ for protons (upper black curves) and $G_{e}(p)$ for electrons (lower red curves), and the time-integrated spectrum of escaped protons, $\Phi_{\text {esc }}$ (blue curves), are given in arbitrary units. Note that $K_{e / p}=0.1$ is adopted here only for clarity.

$d t \approx\left(3 /\left(u_{0}-u_{2}\right)\right)\left(\kappa_{0} / u_{0}+\kappa_{2} / u_{s}\right)(d p / p)$, is reduced only about a factor of two or so from that for the case without MFA.

With our MFA model, the maximum proton momentum at the shock age $t$ can be estimated approximately by

$$
\frac{\left(p_{\mathrm{p}, \max }\right)_{\text {age }}}{m_{p} c} \approx \frac{\sigma_{2}-1}{3 \kappa_{n} \sigma_{2}\left[1+\sigma_{2}\left(B_{0} / B_{2}\right)\right]} \int_{t_{i}}^{t} u_{s}^{2} d t,
$$

where the adiabatic cooling due to spherical expansion is ignored (Kang et al. 2012). Since the simulation follows the ST stage $\left(u_{s} \propto t^{-3 / 5}\right)$ from $t_{i} / t_{0}=0.2$, $p_{\mathrm{p}, \max }$ limited by the shock age increases with time and asymptotes to a maximum value as

$$
\frac{\left(p_{\mathrm{p}, \max }\right)_{\mathrm{age}}}{m_{p} c} \approx 0.35 \frac{\sigma_{2}-1}{\left(\kappa_{n} / \kappa_{o}\right) \sigma_{2}}\left[(0.2)^{-1 / 5}-\left(t / t_{o}\right)^{-1 / 5}\right]
$$

where $\kappa_{o}=u_{o} r_{o}$ and $\sigma_{2}\left(B_{0} / B_{2}\right) \ll 1$ is assumed. Again note that this estimate does not increase with the MFA factor, $B_{1} / B_{0}$. For W1-W4 models with $\sigma_{2} \approx$ $5,\left(p_{\mathrm{p}, \max }\right)_{\mathrm{age}} \approx 5.4 \times 10^{5}\left[(0.2)^{-1 / 5}-\left(t / t_{o}\right)^{-1 / 5}\right] m_{p} c$, which asymptotes to $7.5 \times 10^{5} m_{p} c$ at large $t$.

With our FEB condition, $p_{\mathrm{p}, \max }$ is also limited by the size condition, $\kappa\left(p_{\mathrm{p}, \max }\right) / u_{s} \lesssim \zeta r_{s}$, where again $B_{0}$ should be used to calculate the diffusion coefficient for $p_{\mathrm{p}, \max }$. So the size-limited maximum proton momentum should decrease roughly as

$$
\frac{\left(p_{\mathrm{p}, \max }\right)_{\mathrm{FEB}}}{m_{p} c} \approx \frac{0.53}{\left(\kappa_{n} / \kappa_{o}\right)} \zeta\left(t / t_{o}\right)^{-1 / 5}
$$




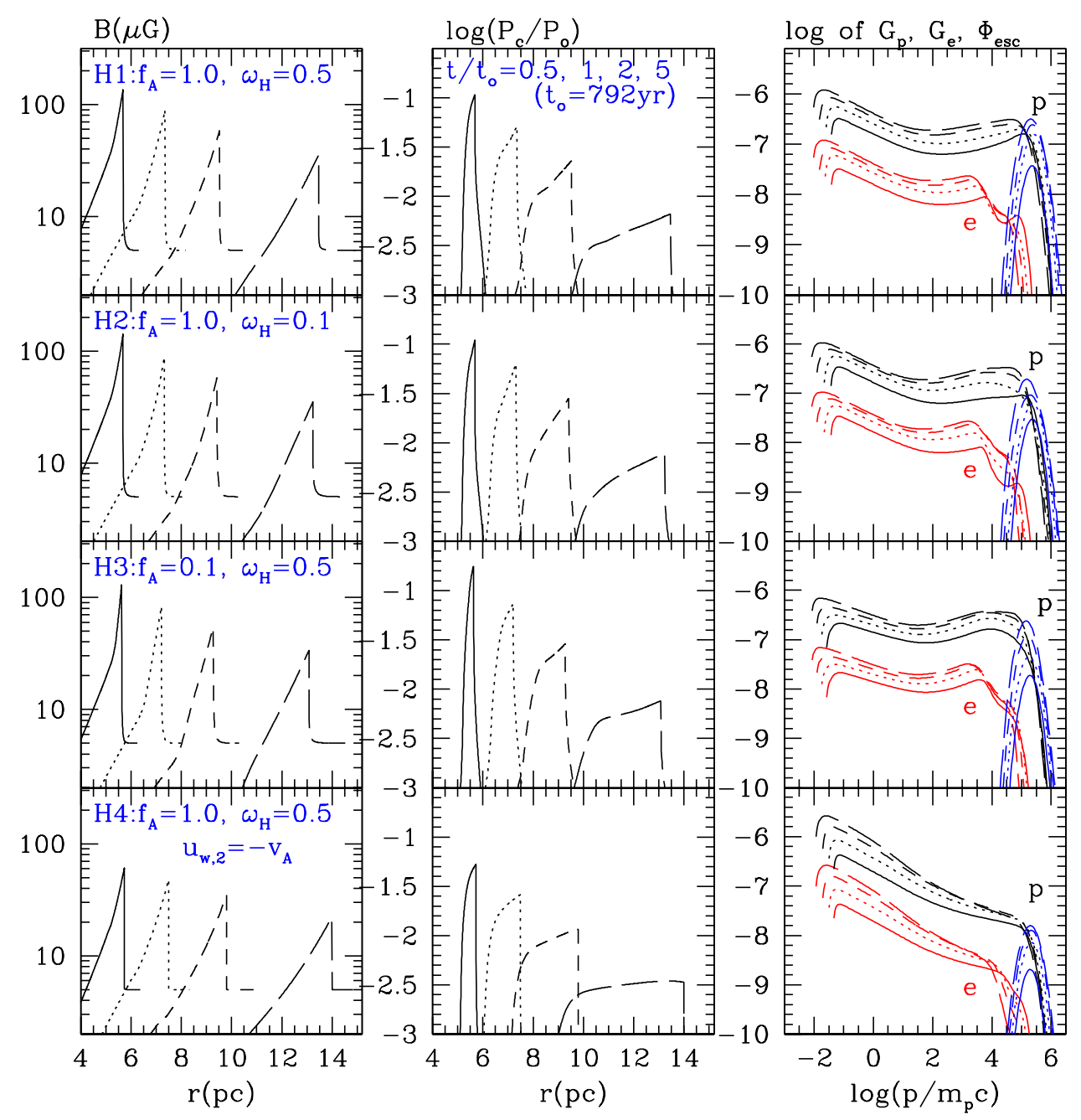

Fig. 2. - Same as Fig. 1 except the results of H1-H4 models are shown.

which does not increase with the MFA factor. For W1$\mathrm{W} 4$ models with $\zeta=0.1,\left(p_{\mathrm{p}, \max }\right)_{\mathrm{FEB}} \approx 10^{5}\left(t / t_{o}\right)^{-1 / 5} m_{p} c$, and for $t / t_{o} \gtrsim 1, p_{\mathrm{p}, \max }$ is limited by the FEB and decreases with time.

On the other hand, the maximum electron momentum can be estimated from the instantaneous properties of the shock,

$$
\frac{p_{\mathrm{e}, \max }}{m_{p} c} \approx 2 \times 10^{4}\left(\frac{B_{1}(t)}{60 \mu \mathrm{G}}\right)^{-1 / 2}\left(\frac{u_{s}(t)}{3000 \mathrm{~km} \mathrm{~s}^{-1}}\right) .
$$

This is derived from the equilibrium condition that the DSA momentum gains per cycle are equal to the synchrotron/iC losses per cycle (Kang et al. 2012). Considering that $B_{1} \propto u_{s}$, the maximum electron momentum limited by cooling scales as $\left(p_{\mathrm{e}, \max }\right)_{\mathrm{cool}} \propto u_{s}^{1 / 2} \propto t^{-3 / 10}$ and so decreases as the shock slows down.

In addition, the volume integrated electron spectrum steepens by one power of $p$ due to radiative cool- ing above the following break momentum,

$$
\frac{p_{\mathrm{e}, \mathrm{br}}(t)}{m_{p} c} \approx 3.1 \times 10^{3}\left(\frac{t}{300 \mathrm{yr}}\right)^{-1}\left(\frac{B_{\mathrm{eff}, 2}}{120 \mu \mathrm{G}}\right)^{-2},
$$

which depends only on the postshock magnetic field strength and the shock age (Kang et al. 2012).

\section{DSA SIMULATION RESULTS}

Figs. 1 and 2 show the spatial profiles of the magnetic field strength and CR pressure, and volumeintegrated distribution functions of protons and electrons, $G(p)=4 \pi \int g(r, p) r^{2} d r$, and the time-integrated spectrum of escaped protons, $\Phi_{\mathrm{esc}}$, for models W1-W4 and $\mathrm{H} 1-\mathrm{H} 4$, respectively. The shock flow structure is modified moderately with $\sigma_{2} \approx 6$ by the CR pressure feedback, except W2 and W3 models in which $\sigma_{2}$ increases up to 10 . The shock modification is weaker for H1-H4 models, because the sonic Mach number is lower due to the hotter ambient gas.

As the CR pressure increases in the shock precursor, 


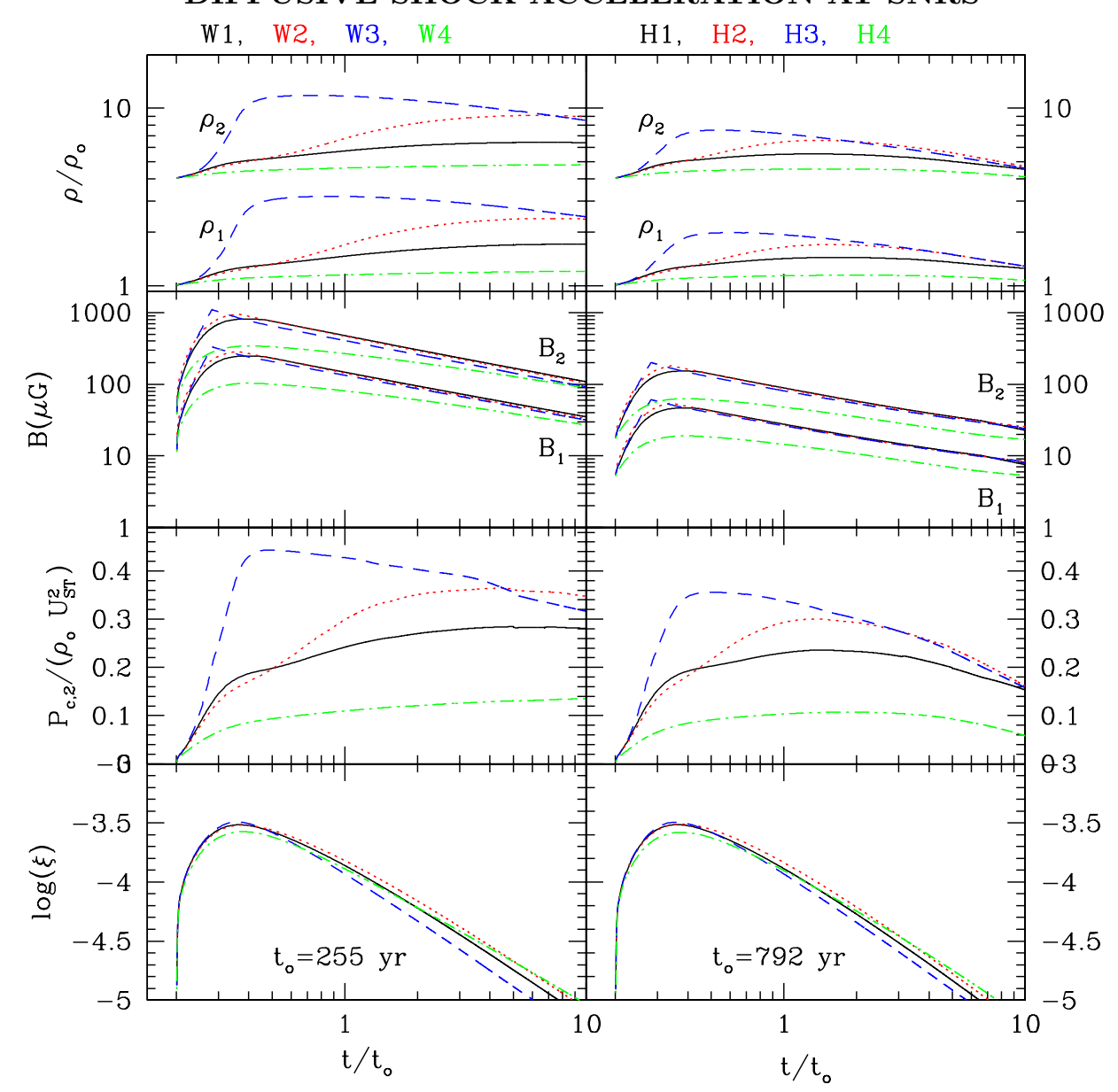

Fig. 3. - Time evolution of the gas density $\rho_{1}\left(\rho_{2}\right)$ immediate upstream (downstream) of the subshock, and magnetic field strengths, $B_{1}$ and $B_{2}$, postshock CR pressure, $P_{c, 2}$ in units of the ram pressure of the unmodified Sedov-Taylor solution, and the injection efficiency, $\xi$, in different models: W1 (black solid lines), W2 (red dotted), W3 (blue dashed), W4 (green dot-dashed) in the left column, and H1 (black solid lines), H2 (red dotted), H3 (blue dashed), H4 (green dot-dashed) in the right column. Note $t_{o}=255 \mathrm{yr}$ for W1-W4 models, while $t_{o}=792 \mathrm{yr}$ for H1-H4 models.

the magnetic fields are amplified and then the CR acceleration saturates due to Alfvénic drift. Since the far upstream Alfvénic Mach number, $M_{A, 0}$, is high, the selfamplified magnetic field strengths, based on the model in Eq. 4, increase up to $B_{1} \sim 200 \mu \mathrm{G}$ in W1-W3 models and to $B_{1} \sim 100 \mu \mathrm{G}$ in W4 models at $t \approx 255 \mathrm{yr}$. These field strengths seem too strong, compared to those inferred from observation $\left(B_{1} \sim 30-50 \mu \mathrm{G}\right)$. As mentioned in Section 2.5, the injection rate realized with our thermal leakage model with $\epsilon_{B}=0.23$ leads to the DSA efficiency in the saturation limits. If one adopts a smaller value of $\epsilon_{B}$, the flow modification and the MFA factor in the precursor could be reduced to the levels more consistent with observations. But we do not attempt to fine-tune the injection parameter to obtain more realistic magnetic field strength, since our focus here is to explore the effects of various wave-particle interactions.

Basically, stronger magnetic fields can lead to faster DSA due to smaller $\kappa$, but faster Alfvénic drift slows down the CR acceleration and softens the CR spectra. So the outcomes of efficient MFA depend nonlinearly on these two opposite effects. Also as the shock slows down in time, $M_{A, 0}$ decreases, so the MFA factor and the significance of Alfvénic drift diminish as well. Thus the behaviors of the different models can be understood mainly by the nonlinear interplay between MFA and Alfvénic drift. The precursor heating by wave dissipation is another important factor, since the CR acceleration is more efficient for stronger subshock with the cooler precursor gas (e.g., W2 model).

As discussed in Section 2.8, the maximum proton momentum reaches only to $p_{\mathrm{p}, \max } \sim 10^{5} \mathrm{~m}_{p} c$ at $t / t_{o} \approx 0.5$ and decreases afterward because of the FEB condition (see also Fig. 5). As the shock slows down, the mean energy of escaping particles decreases and the spectrum of escaped protons, $\Phi_{\text {esc }}$, shifts to lower momentum. For electrons, the maximum momentum, $p_{\text {e,max }}$, decreases with time, as shown in Eq. 16. The volume integrated electron energy spectrum, $G_{e}(p)$, steepens approximately by one power of the momentum due to radiative cooling above the break momentum, $p_{\mathrm{e}, \mathrm{br}}(t)$, which also shifts to lower momentum in time. For example, $p_{\mathrm{e}, \mathrm{br}}(t)$ decreases from $10^{2.8} m_{p} c$ to 


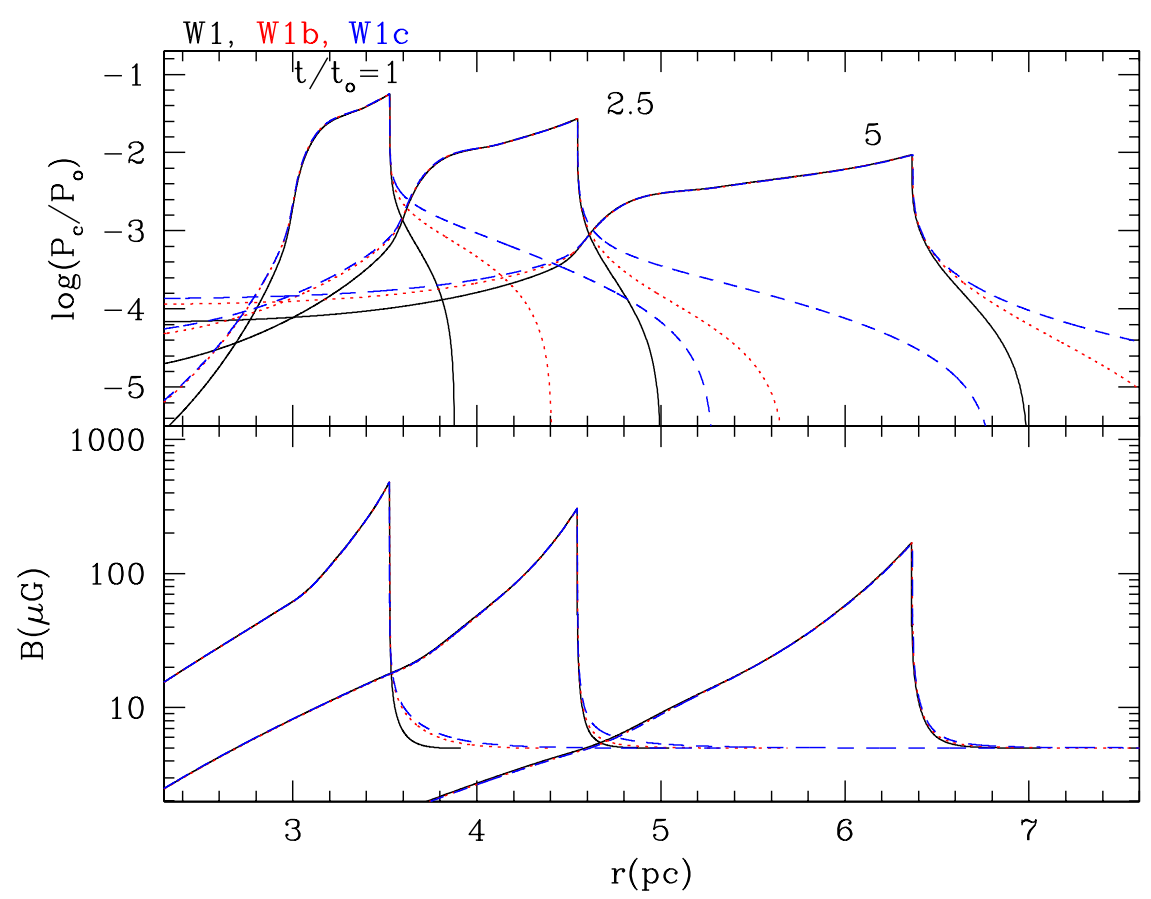

Fig. 4. - The CR pressure (top) and magnetic field strength (bottom) are shown at $t / t_{o}=1,2$, and 5 for W1 model with $r_{\mathrm{FEB}} / r_{s}=1.1$ (solid lines), W1b with $r_{\mathrm{FEB}} / r_{s}=1.25$ (dotted) and W1c with $r_{\mathrm{FEB}} / r_{s}=1.5$ (dashed).

$10^{2.5} m_{p} c$ for $t / t_{o}=0.5-5$ in $\mathrm{W} 1$ model. The peak of $G_{e}(p)$ near $p_{\mathrm{e}, \mathrm{max}}$ comes from the electron population in the upstream region, which cools much less efficiently due to weaker magnetic field there (Edmon et al. 2011). Because of the same reason, the X-ray synchrotron emission due to this population of upstream electrons would be much less pronounced, compared to the electron energy spectrum.

Figs. 1 and 2 demonstrate that the energy spectra of CR protons and electrons, especially near the high energy cutoff, depend on the time-dependent evolution of MFA, the strength of Alfvénic drift, the degree of wave dissipation and heating in addition to the shock dynamics and CR injection history. With our MFA model that depends on the precursor modification, upstream Alfvénic drift affects lower energy particles more strongly, steepening the low energy end of the spectrum more than the high energy end. Because the SNR shock expands spherically and slows down, the shock dynamics and the $\mathrm{CR}$ injection momentum and rate change with time. As a result, the CR proton spectrum still retains the concave curvature. Although upstream Alfvénic drift $\left(u_{w, 1}=+v_{A}\right)$ softens the CR spectra somewhat, downstream Alfvénic drift $\left(u_{w, 2}=-v_{A}\right)$ seems necessary in order to obtain the CR proton spectrum at the high energy end as steep as $E^{-2.3}$ (W4 and H4 models).

Fig. 3 shows the time evolution of the various shock properties for the different models: the density compression factors, amplified magnetic field strengths, postshock CR pressure, and CR injection fraction for W1-W4 models (left column) and H1-W4 models (right column). Compared to the fiducial models (W1 and H1, solid lines), W2 and H2 models (dashed lines) with a lower wave dissipation rate $\left(\omega_{H}=0.1\right)$ have slightly higher magnetic fields and colder gas in the precursor. Both of these effects lead to higher CR pressure, stronger shock modification, and more concave CR spectra. W3 and H3 models (long dashed lines) have a smaller Alfvén speed $\left(f_{A}=0.1\right)$, so the Afvénic drift effects are much weaker, the DSA is more efficient, and the shock structure is more significantly modified. This stronger flow modification leads to higher MFA, so the magnetic field strength increases above the level that should be limited by the energy requirement given by the relation (5). This can be seen in the second row from the top in Fig. 3. In W4 and H4 models (dotdashed lines), the DSA is less efficient due to downstream Alfvénic drift, so the shock flow is less modified and the MFA factor is smaller.

For H1-H4 models both $M_{s}$ and $M_{A, 0}$ are smaller, compared to those for W1-W4 models, so the DSA efficiency is somewhat lower while MFA is much weaker. For example, in H1-H3 models $B_{1} \approx 45 \mu \mathrm{G}$ and $B_{2} \sim 145 \mu \mathrm{G}$ at $t \approx 300 \mathrm{yr}$.

In Figs. 4 and 5 , three models with the different location of FEB are shown: $\zeta=0.1,0.25$, and 0.5 for $\mathrm{W} 1$, W1b, and W1c models, respectively. Fig. 4 shows that the overall distribution of $P_{c}$ is about the same in the three models except far upstream near $r_{\mathrm{FEB}}$, where $P_{c}$ is dominated by the highest energy particles that diffuse over to the FEB location. The precursor structure is determined by the CR pressure distribution, which spreads over the diffusion length of particles that make 


\section{DIFFUSIVE SHOCK ACCELERATION AT SNRS}

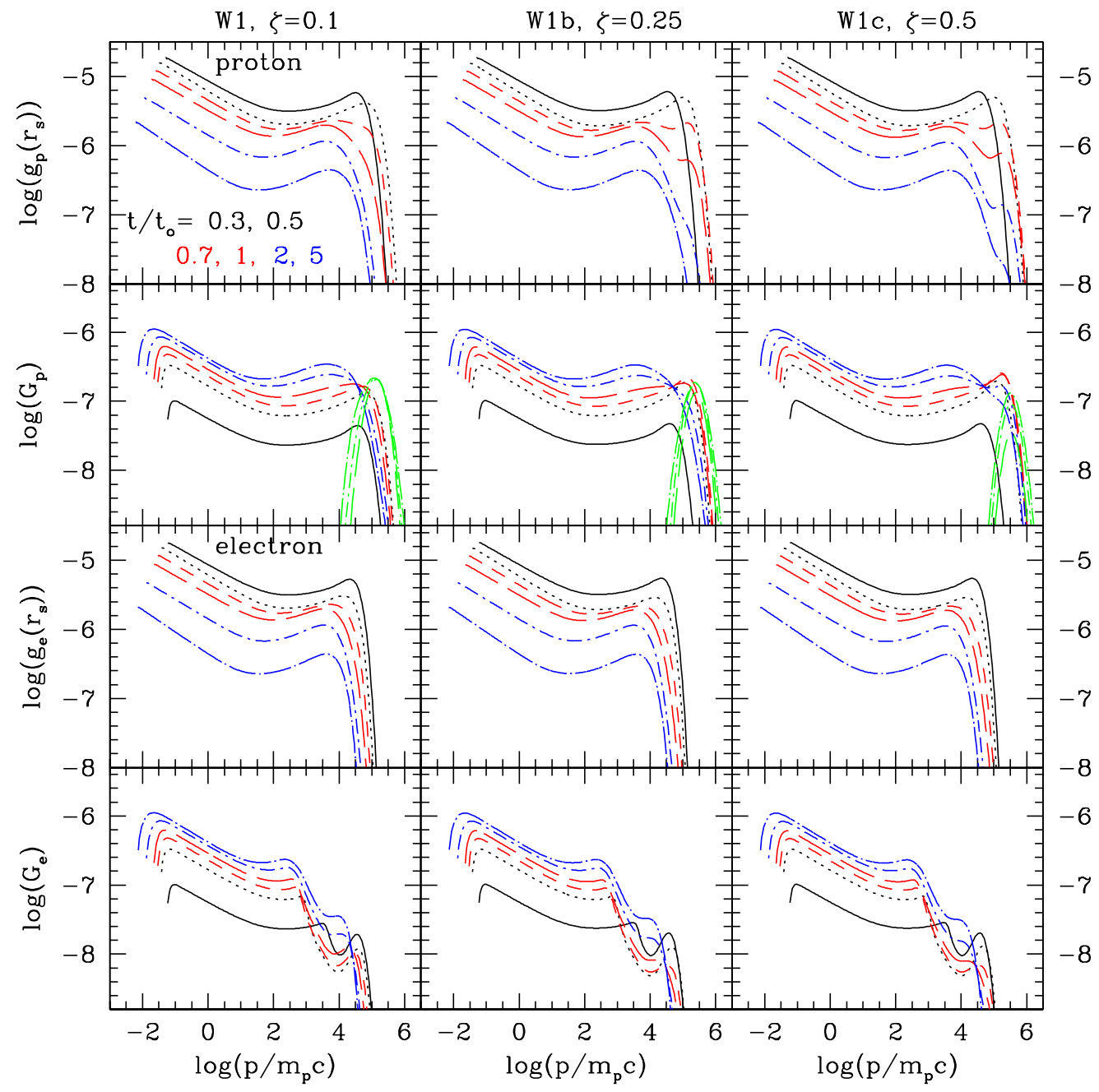

Fig. 5. - Time evolution of the CR spectra for W1 model with $r_{\mathrm{FEB}} / r_{s}=1.1$ (left column), W1b with $r_{\mathrm{FEB}} / r_{s}=1.25$ (middle column) and W1c with $r_{\mathrm{FEB}} / r_{s}=1.5$ (right column). From top to bottom: the distribution function at the shock location for protons, $g_{p}\left(r_{s}\right)$, the volume integrated distribution function for protons, $G_{p}$, the distribution function at the shock location for electrons, $g_{e}\left(r_{s}\right)$, and the volume integrated distribution function for electrons, $G_{e}$, are shown at $t / t_{o}=0.3$ (black solid lines), 0.5 (black dotted), 0.7 (red dashed), 1 (red long dashed), 2 (blue dot-dashed), 5 (blue dot-long dashed). In the second row from the top, the time-integrated spectrum of escaped protons, $\Phi_{\text {esc }}$ is also plotted for $t / t_{o}=11$ (green long dashed lines), 2 (green dot-dashed), 5 (green dot-long dashed).

the greatest contribution to $P_{c}$. As can be seen from the magnetic field profile, the magnetic field precursor width, $L_{\text {pre,B }}$, is about $5-10 \%$ of the shock radius. It is broader for larger $\zeta$ at $t / t_{o}=1$, but later it becomes similar in all three models. Since $L_{\text {pre,B }}$ is smaller than the FEB width, $\zeta r_{s}$, for W1b and W1c models, the acceleration slows down above a certain momentum, when the most energetic particles diffuse beyond the magnetic field precursor where $B(r)$ becomes $B_{0}$ (see Section 2.8).

As shown in Fig. 5, the CR proton spectrum at the shock, $g_{p}\left(r_{s}\right)$, exhibits a concave curvature as a consequence of momentum dependent diffusion across the precursor and slowing-down of the spherical shock. Softening of the spectrum at the low energy end is further enhanced by Alfvénic drift and weakening of the subshock strength. From the age of $t / t_{o} \gtrsim 0.5$ the proton spectrum near the high energy end begins to be affected by FEB and the proton spectrum cuts off at gradually lower $p$ afterward. In W1b and W1c models, slowing down of DSA due to the magnetic field profile in the precursor imprints a feature around $p / m_{p} c \sim 10^{3.5}$ for $t / t_{o} \gtrsim 0.7$, which is not pronounced in W1 model. So the spectral shape of $g_{s}(p)$ at the high energy end becomes complex and its evolution is rather nonlinear. The volume integrated spectrum, $G_{p}$, is relatively smooth and could be steeper than $E^{-2}$ near the high energy cutoff. Also the concavity of $G_{p}$ is much less pronounced than that of $g_{p}\left(r_{s}\right)$.

As expected, more particles escape from the shock in the model with the FEB location closer to the shock (i.e., smaller $\zeta$ ). So the time-integrated number of es- 


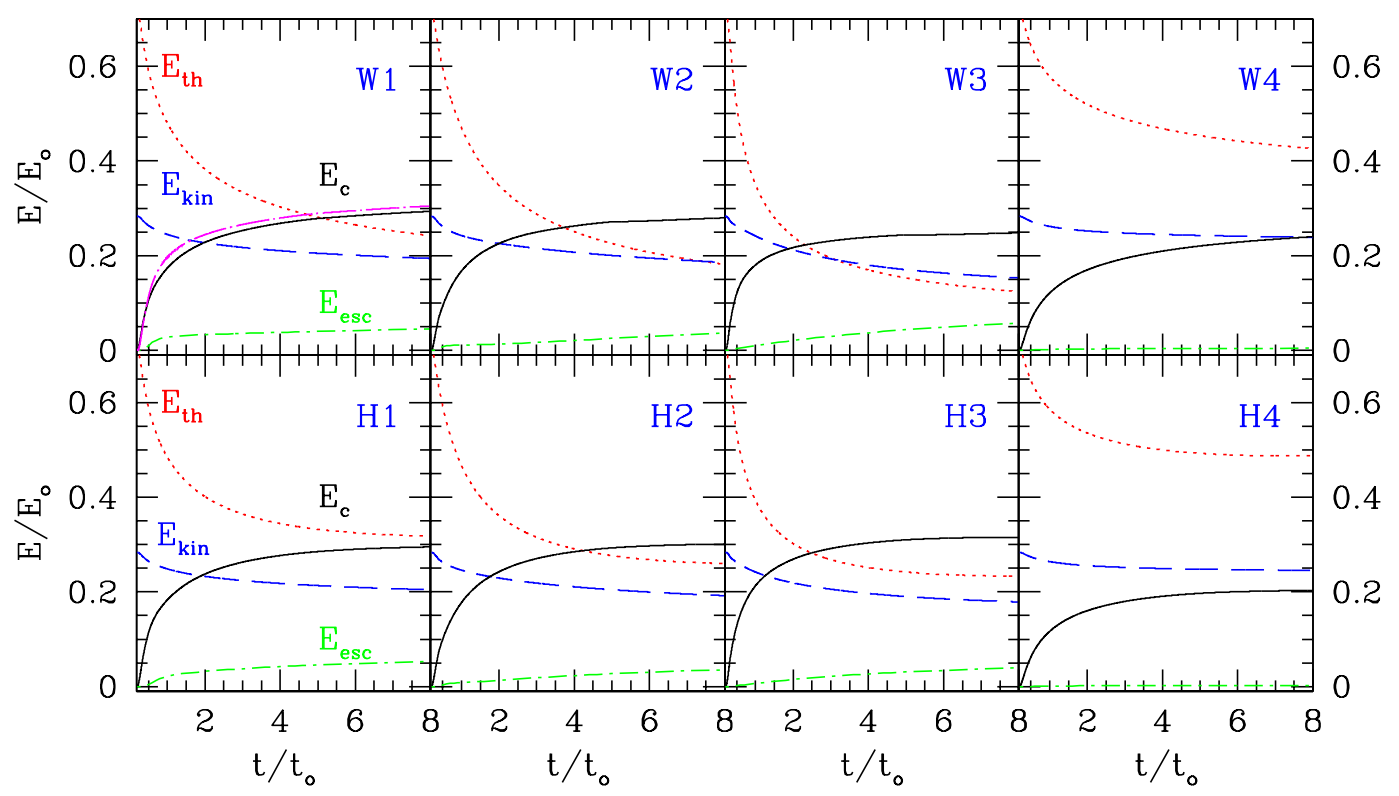

Fig. 6. - Integrated thermal ( $E_{\mathrm{th}}$, red dotted lines), kinetic ( $E_{\mathrm{kin}}$, blue dashed lines), and CR ( $E_{\mathrm{c}}$, black solid lines) energies inside the simulation volume are shown in units of the explosion energy $E_{o}$ for W1-W4 (top) and H1-H4 (bottom) models. In the panel for W1 model, the CR energy for W1c model (magenta dot-long dashed line) is also shown. The green dot-dashed lines represent the fraction of energy carried by the highest energy protons escaped through the FEB. Note $t_{o}=255 \mathrm{yr}$ for W1-W4 models, while $t_{o}=792 \mathrm{yr}$ for H1-H4 models.

caped protons, $\Phi_{\mathrm{esc}}(p)$, is the greatest in $\mathrm{W} 1$ model. This figure demonstrates how the CR proton spectrum is influenced by the time-dependent MFA and escape of the highest energy particles.

On the other hand, the high energy end of the electron spectra (both $g_{e}\left(r_{s}\right)$ and $G_{e}$ ) are not affected by FEB, instead they are limited by the cooling rate and so by the evolution of $B(r, t)$. So it is unlikely that the spectral shape of X-ray synchrotron would carry any information regrading escape of the highest energy protons.

Fig. 6 compares how different energy components $\left(E_{\mathrm{th}}, E_{\mathrm{kin}}, E_{\mathrm{c}}, E_{\mathrm{esc}}\right)$ change in time in the different models. About $30 \%$ of the SN explosion energy is transferred to CRs in the models, in which Alfvénic drift is ignored in the postshock flow (W1-W3 and H1-H3). Slightly less energy, 20-25\%, goes to CRs in the models with downstream Alfvénic drift (W4 and H4 models). The amount of the CR energy generated by SNRs is smaller than what had been suggested by previous DSA simulation studies, in which Alfvénic drift was not included or the Alfvén speed in the background field $\left(B_{0}\right)$ was adopted (e.g., Berezhko \& Völk 1997; Berezhko et al. 2009; Kang \& Jones 2006). Even though the CR acceleration efficiency is reduced, the estimated values, $20-30 \%$ of the SN explosion energy, are still sufficient enough to explain the CR energy escaping from the Galaxy.

Both W1b (not shown here) and W1c (magenta dotlong dashed line) models have the energy components similar to those in W1 model. So the total CR energy generated at SNRs is not significantly affected by the different FEB location, although the high energy end of the proton spectrum is strongly influenced. This is because the amount of energy carried by escaped protons is relatively small. The fraction of escaped CR energy, $E_{\text {esc }}$, is greater in the models with higher CR acceleration efficiency, ranging $0.2-6 \%$ (see green dotdashed lines in Fig. 6). We note that the total energy, $E_{\text {tot }}=E_{\mathrm{th}}+E_{\mathrm{kin}}+E_{\mathrm{c}}+E_{\mathrm{B}}+E_{\mathrm{esc}}$, is not conserved in the simulations, because the magnetic field energy $\left(E_{\mathrm{B}}\right)$ is assumed to decay behind the shock according to the phenomenological model given in Eq. 7. However, the lack of self-consistent treatments of MFA and wave dissipation should have only minor effects on the general conclusions of this study.

\section{SUMMARY}

Observational evidence for nonlinear amplification of magnetic fields at SNR shocks and theoretical studies of wave-particle interactions using plasma and MHD simulations have demonstrated that a detailed understanding of plasma physical processes is essential in testing the DSA hypothesis for the origins of high energy cosmic rays (e.g., Reynolds et al. 2012; Schure et al. 2012, for reviews). In this study, we have explored how some of plasma interactions expected to operate at collisionless shocks affect the outcomes of nonlinear DSA at the outer shock of Type Ia SNRs. First, we adopted phenomenological models for the fol- 
lowing processes: magnetic field amplification via $\mathrm{CR}$ streaming instabilities, Alfvénic drift in the amplified fields both upstream and downstream of the shock, wave damping and heating of the gas, and escape of the highest energy particles through an upstream free escape boundary. Using the kinetic DSA simulations, the time-dependent evolution of the pitch-angle averaged phase-space distribution functions of CR protons and electrons are followed along with the dynamical evolution of Sedov-Taylor blast wave.

According to the recipe for MFA given in Eq. 4 (Caprioli 2012), the amplification factor, $B / B_{0}$, scales with the far upstream Alfvénic Mach number, $M_{A, 0}$, and the degree of the velocity modification in the precursor. So the magnetic field strength is fixed as the background value, $B_{0}$, at the FEB location, increases to $B_{1}$ immediately upstream of the shock, jumps to $B_{2}$ immediately downstream of the shock, and decreases as $B(r)=B_{2}\left(\rho / \rho_{2}\right)$ behind the shock. Since the spatial profile of $B(r)$ is not a simple step function (which is valid for steady-state planar shocks), the time-dependent history of the particle acceleration becomes nonlinear and much more complex. For example, the highest energy protons diffuse over longer length scales in the precursor as the momentum increases, so the diffusion length $\left(l_{\text {diff }} \propto p_{\mathrm{p}, \max } / B\right)$ increases not only because of higher $p_{\mathrm{p} \text {, max }}$ but also because of lower $B$ near the FEB location. As a result, the acceleration slows down gradually at a certain $p$ above which the CR spectrum becomes softer, so the maximum energy does not scale linearly with the MFA factor. This contradicts the common expectation that the maximum energy of CR ions, $E_{\max } \propto B \cdot Z$, can increase up to $10^{15.5} Z \mathrm{eV}$, if the upstream magnetic field is amplified by a factor of 10 or so (e.g., Hillas 2005). To accelerate the particles to such high energies, the turbulent magnetic fields should be amplified on the diffusion length scale of $\kappa\left(p_{\mathrm{p}, \max }\right) / u_{s} \sim r_{g}\left(p_{\mathrm{p}, \max }\right)\left(c / u_{s}\right)$, which is much larger than the gyro radius, $r_{g}\left(p_{\mathrm{p}, \max }\right)$, itself.

Drift of scattering centers away from the shock (both upstream and downstream) is thought to steepen the CR spectrum and to lead to less efficient acceleration (Bell 1978; Zirakashvili \& Ptuskin 2008; Caprioli 2012). The interplay between MFA and Alfvénic drift is nonlinear: higher magnetic field means smaller scattering lengths and faster acceleration, while faster Alfvénic drift leads to steeper spectrum and slower acceleration. With our model for fast Alfvénic drift in the amplified magnetic field precursor, for Bohm-like diffusion $(\kappa \propto p)$, the low energy end of the CR spectrum is more strongly steepened, compared to the high energy end. So at strong CR modified shocks the CR spectrum is expect to retain the concave curvature and its spectral shape is governed by the time-dependent evolution of $B(r, t)$, which in turn depends on the velocity modification in the precursor (Kang 2012). Moreover, as the spherically expanding blast wave slows down in time, the $\mathrm{CR}$ injection momentum $\left(p_{\text {inj }}\right)$ and rate $\left(\xi n_{0} u_{s}\right)$ decrease and the CR population cools adiabatically.
Since the high energy end of the CR proton spectrum consists of the particles that are injected during the early stages of SNRs, the spectral shape near the high energy cutoff can be estimated only by following the time-dependent evolution of the CR injection and MFA as well as the dynamical evolution of the SNR shock. Damping of waves far upstream and resulting escape of the highest energy particles also regulate the high energy end of the CR spectrum. For the higher rate of wave damping (larger $\omega_{H}$ ), the subshock is weaker due to heating of the background plasma in the precursor, leading to less efficient DSA. We also explored a simple FEB condition, in which $f(r, p)=0$ for $r \geq r_{\mathrm{FEB}}=\zeta r_{s}$, where $\zeta=0.1-0.5$ is considered. We demonstrated that the $\mathrm{CR}$ proton spectrum near the high energy cutoff is strongly influenced by the FEB location, and in fact $p_{\mathrm{p} \text {,max }}$ is likely to be limited by particle escape rather than the shock age. Thus it is important to understand how the MHD turbulence is amplified and dissipated through wave-particle interactions on the scales larger than the CR gyroradius.

Previous studies of DSA at SNRs showed that up to $60 \%$ of the SN explosion energy can be transferred to the CR components (Berezhko \& Völk 1997; Berezhko et al. 2009; Kang \& Jones 2006). With the fast Afvénic drift in the amplified fields, we find that DSA can convert about $20-30 \%$ of the explosion energy into CRs for the models considered here. Yet this should be sufficient to replenish escaping CRs from the Galaxy with a luminosity of $L_{C R} \approx 10^{41} \mathrm{erg} \mathrm{s}^{-1}$.

This study demonstrates that a detailed understanding of plasma physical processes operating at collisionless shocks is crucial in predicting the CR energy spectra accelerated at SNR shocks and nonthermal emissions due to those CRs. We will present elsewhere the results of nonthermal radiation spectrum, which is estimated through post processing of the DSA simulation data obtained in this study.

\section{ACKNOWLEDGMENTS}

This work was supported by a 2-Year Research Grant of Pusan National University. The author thanks T. W. Jones, D. Capriloi and the anonymous referee for the constructive suggestions and comments to the paper. The author also thanks Vahe Petrosian and KIPAC for their hospitality during the sabbatical leave at Stanford University where a part of the paper was written.

\section{REFERENCES}

Abdo, A. A., et al. 2010, Fermi Large Area Telescope Observations of the Supernova Remnant W28 (G6.40.1), ApJ, 718, 348

Acero, F., et al. 2010, First Detection of VHE $\gamma$-Rays from SN 1006 by HESS, A\&A, 516, A62

Acciari, V. A., et al. 2011, Discovery of TeV GammaRay Emission from Tycho's Supernova Remnant, ApJ, 730, L20 
Bamba, A., Yamazaki, R, Ueno, M., \& Koyama, K. 2003, Small-Scale Structure of the SN 1006 Shock with Chandra Observations, ApJ, 589, 827

Bell, A. R. 1978, The Acceleration of Cosmic Rays in Shock Fronts. I, MNRAS, 182, 147

Bell, A. R. 2004, Turbulent Amplification of Magnetic Field and Diffusive Shock Acceleration of Cosmic Rays, MNRAS, 353, 550

Beresnyak, A., Jones, T. W., \& Lazarian, A. 2009, Turbulence-Induced Magnetic Fields and Structure of Cosmic Ray Modified Shocks, ApJ, 707, 1541

Berezhko, E. G., \& Völk, H. J. 1997, Kinetic Theory of Cosmic Rays and Gamma Rays in Supernova Remnants. I. Uniform Interstellar Medium, Astropart. Phys., 7, 183

Berezhko, E. G., Ksenofontov, L. T., \& Völk, H. J. 2009, Cosmic Ray Acceleration Parameters from Multi-Wavelength Observations. The Case of SN 1006, A\&A, 505, 169

Berezhko, E. G., Ksenofontov, L. T., \& Völk, H. J. 2012, Nonthermal Emission of Supernova Remnant SN 1006 Revisited: Theoretical Model and the H.E.S.S. Results, ApJ, 759, 12

Blandford, R. D., \& Eichler, D. 1987, Particle Acceleration at Astrophysical Shocks - a Theory of CosmicRay Origin, Phys. Rept., 154, 1

Bykov, A. M., Osipov, S. M., \& Ellison, D. C. 2011, Cosmic Ray Current Driven Turbulence in Shocks with Efficient Particle Acceleration: the Oblique, Long-Wavelength Mode Instability, MNRAS, 410, 39

Caprioli, D. 2011, Understanding Hadronic GammaRay Emission from Supernova Remnants, JCAP, 5, 26

Caprioli, D. 2012, Cosmic-Ray Acceleration in Supernova Remnants: Non-Linear Theory Revised, JCAP, 7,38

Caprioli, D., Amato, E., \& Blasi, P. 2010, NonLinear Diffusive Shock Acceleration with FreeEscape Boundary, Astropart. Phys., 33, 307

Caprioli, D., Blasi, P., Amato, E., \& Vietri, M. 2009, Dynamical Feedback of Self-Generated Magnetic Fields in Cosmic Ray Modified Shock, MNRAS, 395, 895

Caprioli, D., \& Spitkovsky, A. 2012, Cosmic-RayInduced Filamentation Instability in Collisionless Shocks, arXiv:1211.6765

Drury, L. O'C. 1983, An Introduction to the Theory of Diffusive Shock Acceleration of Energetic Particles in Tenuous Plasmas, Rept. Prog. Phys., 46, 973

Drury, L. O'C., \& Downes, T. P. 2012, Turbulent Magnetic Field Amplification Driven by Cosmic Ray Pressure Gradients, MNRAS, 427, 2308
Drury, L. O'C. 2011, Escaping the Accelerator: How, When and in What Numbers Do Cosmic Rays Get out of Supernova Remnants?, MNRAS, 415, 1807

Edmon, P. P., Kang, H., Jones, T. W., \& Ma, R. 2011, Non-Thermal Radiation from Type Ia Supernova Remnants, MNRAS, 414, 3521

Eriksen, K. A., Hughes, J. P., Badenes, C., et al. 2011, Evidence for Particle Acceleration to the Knee of the Cosmic Ray Spectrum in Tycho's Supernova Remnant, ApJ, 728, L28

Gargaté, L., Fonseca, R. A., Niemiec, J., Pohl, M., Bingham, R., \& Silva, L. O. 2010, The Nonlinear Saturation of the Non-resonant Kinetically Driven Streaming Instability, ApJ, 711, L127

Gargaté, L., \& Spitkovsky, A. 2012, Ion Acceleration in Non-Relativistic Astrophysical Shocks, ApJ, 744, 67

Giacalone, J., \& Jokipii, J. R. 2007, Magnetic Field Amplification by Shocks in Turbulent Fluids, ApJ, 663, L41

Giordano, F., et al. 2012, Fermi Large Area Telescope Detection of the Young Supernova Remnant Tycho, ApJ, 744, L2

Guo, F., Jokipii, J. R., \& Kota, J. 2010, Particle Acceleration by Collisionless Shocks Containing LargeScale, Magnetic-Field Variations, ApJ, 725, 128

Hillas, A. M. 2005, Can Diffusive Shock Acceleration in Supernova Remnants Account for High Energy Galactic, Cosmic Rays?, Journal of Physics G, 31, R95

Jones, T. W. 1993, Alfvén Wave Transport Effects in the Time Evolution of Parallel Cosmic-Ray-Modified Shocks, ApJ, 413, 619

Kang, H. 2006, Cosmic Ray Acceleration at Blast Waves from Type Ia Supernovae, JKAS, 39, 95

Kang, H. 2010, Cosmic Ray Spectrum in Supernova Remnant Shocks, JKAS, 43, 25

Kang, H. 2012, Diffusive Shock Acceleration with Magnetic Field Amplification and Alfvénic Drift JKAS, 45,127

Kang, H., Edmon, P. P., \& Jones, T. W. 2012, Non-Thermal Radiation from Cosmic-Ray Modified Shocks, ApJ, 745, 146

Kang, H., \& Jones, T. W. 2006, Numerical Studies of Diffusive Shock Acceleration at Spherical Shocks, Astropart. Phys., 25, 246

Kang, H., Jones, T. W., \& Gieseler, U. D. J. 2002, Numerical Studies of Cosmic-Ray Injection and Acceleration, ApJ, 579, 337

Lee, S., Ellison, D. C., \& Nagataki, S. 2012, A Generalized Model of Nonlinear Diffusive Shock Acceleration Coupled to an Evolving Supernova Remnant, ApJ, 750, 156 
Lucek, S. G., \& Bell, A. R. 2000, Non-Linear Amplification of a Magnetic Field Driven by Cosmic Ray Streaming, MNRAS, 314, 65

Malkov, M. A., \& Drury, L. O'C. 2001, Nonlinear Theory of Diffusive Acceleration of Particles by Shock Waves, Rep. Progr. Phys., 64, 429

Malkov, M. A., Diamond, P. H., \& Sagdeev, R. Z. 2011, Mechanism for Spectral Break in Cosmic Ray Proton Spectrum of Supernova Remnant W44, Nature Communications, 2, 194

Mandelartz, M., \& Tjus, J. B. 2013, A Statistical Study of Galactic SNR Source Spectra Detected at $>\mathrm{GeV}$ Energies, arXiv:1301.2437

Morlino, G., Amato, E., \& Blasi, P. 2009, Gamma-Ray Emission from SNR RX J1713.7-3946 and the Origin of Galactic Cosmic Rays, MNRAS, 392, 240

Morlino G., \& Caprioli, D. 2012, Strong Evidence for Hadron Acceleration in Tycho's Supernova Remnant, A\&A, 538, 81

Ohira, Y., Reville, B., Kirk, J. G., \& Takahara, F. 2009, Two-Dimensional Particle-In-Cell Simulations of the Nonresonant, Cosmic-Ray-Driven Instability in Supernova Remnant Shocks, ApJ, 698, 445

Parizot, E., Marcowith, A., Ballet, J., \& Gallant, Y. A. 2006, Observational Constraints on Energetic Particle Diffusion in Young Supernovae Remnants: Amplified Magnetic Field and Maximum Energy, A\&A, 453,387

Ptuskin, V. S., \& Zirakashvili, V. N. 2005, On the Spectrum of High-Energy Cosmic Rays Produced by Supernova Remnants in the Presence of Strong Cosmic-Ray Streaming Instability and Wave Dissipation, A\&A, 429, 755

Ptuskin, V. S., Zirakashvili, V. N., \& Seo, E. 2010, Spectrum of Galactic Cosmic Rays Accelerated in Supernova Remnants, ApJ, 718, 31

Reville, R., \& Bell, A. R. 2012, A Filamentation Instability for Streaming Cosmic Rays, MNRAS, 419, 2433

Reville, R., \& Bell, A. R. 2013, Universal Behaviour of Shock Precursors in the Presence of Efficient Cosmic-Ray Acceleration, arXiv:1301.3173

Reynolds, S. P. 2008, Supernova Remnants at High Energy, ARA\&A, 46, 89

Reynolds, S. P., Gaensler, B. M., \& Bocchino, F. 2012, Magnetic Fields in Supernova Remnants and PulsarWind Nebulae, Space Sci. Rev., 166, 231

Riquelme, M. A., \& Spitkovsky, A. 2009, Nonlinear Study of Bell's Cosmic Ray Current-Driven Instability, ApJ, 694, 626

Riquelme, M. A., \& Spitkovsky, A. 2010, Magnetic Amplification by Magnetized Cosmic Rays in Supernova Remnant Shocks, ApJ, 717, 1054
Rogachevskii, I., Kleeorin, N., Brandenburg, A., \& Eichler, D. 2012, Cosmic-Ray Current-Driven Turbulence and Mean-Field Dynamo Effect, ApJ, 753, 6

Schlickeiser, R. 1989, Cosmic-Ray Transport and Acceleration. II. Cosmic Rays in Moving Cold Media with Application to Diffusive Shock Wave Acceleration, ApJ, 336, 264

Schlickeiser R. 2002, Cosmic Ray Astrophysics (Berlin: Springer)

Schure, K. M., Bell, A. R, Drury, L. O’C., \&. Bykov, A. M. 2012, Diffusive Shock Acceleration and Magnetic Field Amplification, Space Sci. Rev., 173, 491

Skilling, J. 1975, Cosmic Ray Streaming. I - Effect of Alfvén Waves on Particles, MNRAS, 172, 557

Vladimirov, A. E., Bykov, A. M., \& Ellison, D. C. 2008, Turbulence Dissipation and Particle Injection in Nonlinear Diffusive Shock Acceleration with Magnetic Field Amplification, ApJ, 688, 1084

Völk, H. J., Berezhko, E. G., \& Ksenofontov, L. T. 2005, Magnetic Field Amplification in Tycho and Other Shell-Type Supernova Remnants, A\&A, 433, 229

Zirakashvili, V. N., \& Ptuskin, V. S. 2008, Diffusive Shock Acceleration with Magnetic Amplification by Nonresonant Streaming Instability in Supernova Remnants, ApJ, 678, 939

Zirakashvili, V. N., \& Ptuskin, V. S. 2012, Numerical Simulations of Diffusive Shock Acceleration in SNRs, APh, 39, 12 